\title{
Demenz
}

\section{Wirkt Ginkgo-Extrakt protektiv?}

- Ein Extrakt von Ginkgo biloba hat in der Verumgruppe der GuidAge-Studie die Inzidenz von Morbus Alzheimer nicht signifikant gesenkt. Dennoch könnte es sein, dass die langzeitige Einnahme protektiv wirkt.

An der multizentrischen, randomisierten, doppelblinden und placebokontrollierten Studie, die von Forschern des französischen „Institut national de la santé et de la recherche médicale" (Inserm) geleitet wurde, hatten sich über 2800 Frauen und Männer beteiligt. Sie waren über 70 Jahre alt und hatten bei ihrem Hausarzt über Gedächtnisprobleme geklagt. Jeweils rund 1400 Studienteilnehmer erhielten daraufhin zweimal täglich $120 \mathrm{mg}$ standardisierten Ginkgo-biloba-Extrakt (EGb761) oder Placebo. Fünf Jahre lang wurden die Probanden nachbeobachtet. Am Ende des Studienzeitraums hatten knapp 5\% der
Teilnehmer eine Demenz entwickelt statt, wie vorausberechnet, rund $14 \%$. Die statistische Aussagekraft der Studie wurde durch diese Diskrepanz geschwächt. Die Alzheimerinzidenz in der Placebogruppe lag bei 5,2\%, in der Verumgruppe bei 4,3\%. Diese Differenz war statistisch nicht aussagekräftig $(p=0,3)$.

\section{Halbierung des Alzheimer-Risikos}

In einer Post-hoc-Analyse ermittelten die Inserm-Wissenschaftler in der Ginkgo-biloba-Gruppe allerdings nahezu eine Halbierung des Risikos, an Alzheimer zu erkranken - vorausgesetzt, der Extrakt wurde länger als drei Jahre eingenommen ( $p=$ 0,02 ). Für Demenzerkrankungen allgemein betrug die Reduktion 47\%, ebenfalls unter der Maßgabe langzeitiger Ginkgo-Einnahme $(p=0,02)$. Es könne sich bei den Ergebnissen um Zufall handeln, schreiben die
Forscher und verweisen auf die geringe Gesamtzahl von Alzheimererkankungen in der Studie. Allerdings habe bereits eine frühere Studie zur Alzheimerprävention mit nicht steroidalen Antirheumatika darauf hingedeutet, dass sich eine vorbeugende Behandlung im Zeitverlauf unterschiedlich auswirken könnte (Breitner JC et al. Alzheimers Dement 2011; 7: 402-11).

Das letzte Wort zur präventiven Potenz von Ginkgo biloba scheint noch nicht gesprochen zu sein. Die französischen Forscher regen an, speziell die Effekte einer Langzeitexposition gegenüber Ginkgo-Extrakt in künftigen Studien zu klären.

\section{- Dr. Robert Bublak}

Quelle: Vellas B et al. Long-term use of standardised ginkgo biloba extract for the prevention of Alzheimer's disease (GuidAge): a randomized placebo-controlled trial. Lancet Neurol 2012, online 6. September; http://dx.doi.org/10.1016/ S1474-4422(12)70206-5

\section{GuidAge-Studie lässt nur eingeschränkte Aussagen zu}

MMW: Die Autoren der GuidAge-Studie haben ein negatives Fazit gezogen, was die Möglichkeit betrifft, mit Ginkgo biloba der Entstehung eines Morbus Alzheimer vorzubeugen. Sind Sie enttäuscht? Ihl: Nein. Die Rahmenbedingungen der Studie waren nicht geeignet, eine Aussage zur präventiven Wirksamkeit einer Substanz zuzulassen, was die Autoren selbst beschreiben und auch in einem Kommentar von Lon Schneider, Los Angeles, im "Lancet Neurology" anklingt. Leider widersprechen sich alle dann selbst und ziehen ohne hinreichende Datenlage negative Schlüsse.

MMW: Vor allem in der Post-hoc-Analyse räumen die Autoren um Bruno Vellas aber zumindest die Möglichkeit eines präventiven Effekts nach mehr als dreijähriger Ginkgo-Einnahme ein. Halten Sie das für realistisch?

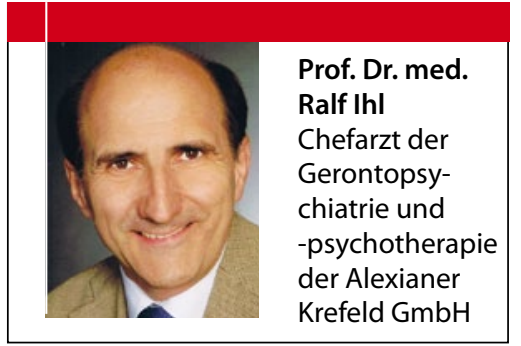

Ihl: Vellas macht dabei auf einen wichtigen Aspekt aufmerksam, den schon frühere Studien gezeigt haben. Wenn man wissen will, ob eine Substanz präventiv wirkt, muss sie auch eingenommen werden. Interessanterweise war eine solche Vorgehensweise im Studiendesign vorgesehen und durchgeführt worden. Sie erbrachte einen positiven Effekt. Gesetzt den Fall, dass diese Größenordnung tatsächlich zutrifft, was aufgrund der Datenlage der Vorstudien EPIDOS, GEM und PAQUID nicht ausgeschlossen ist, wäre es dringend notwendig, die Daten weiterzuverfolgen.

MMW: Was ist Ihrer Meinung nach kritikwürdig an der Studie von Vellas et al.? Ihl: Schneider bezeichnet in seinem Kommentar Präventionsstudien zu Recht als "Pionierarbeit". Da bleiben Fehler nicht aus. Wir gehen heute davon aus, dass Demenzen schon 20-30 Jahre vor der Diagnose entstehen. Es steht damit in Frage, ob es günstig ist, eine Studie in einer Population mit so hohem Durchschnittsalter durchzuführen und nur über einen Zeitraum von wenigen Jahren. Hier ist das Risiko sehr hoch, dass man bei bereits erkrankten Menschen eine Vorbeugung versucht. Das kann naturgemäß eher frustran sein.

Interview: Dr. Robert Bublak 